\title{
El rol del Big data como herramienta de comunicación estratégica y construcción de reputación en Chile
}

\author{
The role of Big Data as a tool for strategic \\ communication and reputation building in Chile \\ O papel do Big Data como ferramenta de comunicação \\ estratégica e construção de reputação no Chile
}

\author{
Juan Cristóbal Portales Echeverría ${ }^{1}$ \\ Universidad Adolfo Ibáñez (Chile) \\ juan.portales@uai.cl \\ Juan Castañeda Alcaíno \\ Universidad Alberto Hurtado (Chile) \\ jcastana@uc.cl
}

Fecha de recepción: 06 de febrero de 2018

Fecha de recepción evaluador: 30 de septiembre de 2018

Fecha de recepción corrección: 12 de noviembre de 2018

\footnotetext{
1 Juan Cristóbal Portales Echeverría es Periodista Universidad Católica de Chile, MSc Political Communication London School of Economics and Political Science y PhD Comunicación Política Universidad Complutense de Madrid. Docente, Investigador y Director Magíster de Comunicación Estratégica, Universidad Adolfo Ibáñez. Ha sido asesor de varios gobiernos, campañas políticas y empresas en Chile, Colombia, Ecuador, República Dominicana y Haití. https://orcid.org/0000-0002-2112-1501

2 Juan Alberto Castañeda Alcaíno es Cientista Político Universidad Católica de Chile, Magíster en Estudios Sociales y Políticos Latinoamericanos U. Alberto Hurtado, Doctor (C) en Sociología por la misma universidad. Docente, Investigador U. Católica Silva Henríquez, Chile. Ha sido docente desde hace más de 10 años. https://orcid.org/0000-0002-2910-6816
} 


\title{
Resumen
}

La presente investigación da cuenta de un proceso escasamente abordado en los estudios de Comunicación como es la gestión de la información al interior de las empresas y el uso, estrategias y gestión con que se abordan a grupos de interés y social media influencers en cuanto canalizadores sociales de marca. La noción de influencia ha vuelto a cobrar importancia en el área de la comunicación estratégica con el auge de las diversas plataformas de información, relacionamiento digital y el avance hacia un contexto de alta competencia y desconfianza hacia las empresas. Al objeto de lograr esa influencia hoy resulta fundamental un uso oportuno del Big Data, definido como un gran volumen y flujo de diferentes formas de datos procedentes de diversas fuentes (externas e internas) en constante procesamiento, que producen nuevos conocimientos. En ese sentido, el big data facilita el acceso a determinados grupos de stakeholders con gran precisión. Pese a esta evidencia, en la presente investigación se constata que los profesionales de la comunicación en Chile no lo ven así. Entienden la comunicación estratégica fundamentalmente no como una vía construir confianza, pero sí para alinear una estrategia corporativa con una estrategia comunicacional y como instrumental para otorgar una orientación mayor a los directivos a la hora de tomar decisiones y definir objetivos estratégicos de negocio. Para abordar nuestro trabajo investigativo, trataremos en primer lugar una discusión teórica acotada al grado de profesionalización de la comunicación estratégica, expresada en la adopción y uso de diversas tendencias y herramientas de práctica, especialmente de análisis de Big Data, para luego identificar las principales características del estudio. Posteriormente presentaremos los resultados de la investigación y nuestras conclusiones. Se constata en el caso de Chile que la mayoría de los profesionales de la comunicación carecen de las competencias y herramientas para obtener altos rendimientos y efectividad a la hora de posicionar las marcas e influir al interior de la sociedad interconectada.

Palabras clave: Big Data, media influencers, comunicación, empresas, sociedad interconectada.

\begin{abstract}
The present investigation accounts for a process scarcely addressed in Communication studies such as the management of information within companies and the use, strategies and management with which they are addressed to interest groups and social media influencers as social channeling branded. The notion of influence has once again become important in the area of strategic communication with the rise of various information platforms, digital relationships and progress towards a context of high competition and distrust of companies. In order to achieve this influence, a timely use of Big Data, defined as a large volume and flow of different forms of data from different sources (external and internal) in constant processing, which produce new knowledge is fundamental. In that sense, big data facilitates access to certain groups of stakeholders with great precision. Despite this evidence, in this research it is found that communication professionals in Chile do not see it that way. They understand strategic communication fundamentally not as a way to build trust, but to align a corporate strategy with a communication strategy and as an instrument to give managers greater guidance when making decisions and
\end{abstract}


defining strategic business objectives. To address our research work, we will first discuss a theoretical discussion limited to the degree of professionalization of strategic communication, expressed in the adoption and use of various trends and practice tools, especially Big Data analysis, to then identify the main characteristics of the study. Later we will present the results of the investigation and our conclusions.In the case of Chile, the majority of communication professionals lack the skills and tools to obtain high performance and effectiveness when positioning brands and influencing the interconnected society.

Keywords: Big Data, media influencers, communication, companies, interconnected society.

\section{Resumo}

A presente investigação explica um processo pouco abordado em estudos de Comunicação, como o gerenciamento de informações dentro de empresas e o uso, estratégias e gerenciamento com os quais eles são direcionados a grupos de interesse e influenciadores de mídias sociais como marca de canais sociais. A noção de influência mais uma vez se tornou importante na área de comunicação estratégica com o surgimento de várias plataformas de informação, relações digitais e progresso em direção a um contexto de alta competição e desconfiança das empresas. A fim de alcançar essa influência, o uso oportuno de Big Data, definido como um grande volume e fluxo de diferentes formas de dados de diferentes fontes (externas e internas) em constante processamento, que produzem novos conhecimentos é fundamental. Nesse sentido, o big data facilita o acesso a determinados grupos de stakeholders com grande precisão. Apesar dessa evidência, nesta pesquisa verifica-se que os profissionais de comunicação no Chile não a veem assim. Eles entendem a comunicação estratégica fundamentalmente não como uma maneira de construir confiança, mas para alinhar uma estratégia corporativa com uma estratégia de comunicação e como um instrumento para dar aos gerentes maior orientação ao tomar decisões e definir objetivos estratégicos de negócios. Para abordar o nosso trabalho de pesquisa, primeiro discutiremos uma discussão teórica limitada ao grau de profissionalização da comunicação estratégica, expressa na adoção e uso de várias tendências e ferramentas práticas, especialmente a análise Big Data, para então identificar as principais características do estudo. . Posteriormente, apresentaremos os resultados da investigação e nossas conclusões. No caso do Chile, a maioria dos profissionais de comunicação não possui as habilidades e ferramentas para obter alto desempenho e eficácia ao posicionar marcas e influenciar a sociedade interconectada.

Palavras-chave: Big Data, influenciadores de mídia, comunicação, empresas, sociedade interconectada. 


\section{Introducción}

El artículo presenta los resultados preliminares de un estudio que realiza la European Public Relations Education and Research Association (EUPRERA), junto con la Asociación de Directivos de Comunicación de España (en adelante Dircom) en conjunto con el Magister de Comunicación estratégica de la Universidad Adolfo Ibañez de Chile (a cargo de su ejecución en este país). Tal estudio midió las habilidades, conocimientos y competencias de los profesionales de la comunicación y empresas en Latinoamérica y Chile en materia de uso de big data, uso de herramientas digitales y entendimiento-aproximación hacia los denominados social media influencers.

Para el caso de este último país, y relacionados con los desafíos que presenta la comunicación moderna a la hora de posicionar el valor de las empresas en una sociedad cada vez más interconectada, los investigadores establecieron como pregunta de investigación base ¿cuáles eran las competencias cognitivas y habilidades de los profesionales de la comunicación en un escenario de proliferación de los big data y uso intensivo de las redes sociales? Derivado de lo anterior, nuestro objetivo central se centró en identificar el nivel de comprensión y penetración de herramientas, estrategias, canales y embajadores funcionales a una creación de valor corporativo y sostenibilidad de largo plazo.

La relevancia de este estudio radica entonces en que mide las habilidades, conocimientos y competencias de los profesionales de la comunicación en materias hasta ahora socializadas como importantes y obvias dentro de una práctica profesional, pero no necesariamente entendidas e incorporadas de manera profesional por los llamados practitioners.

Para abordar nuestro trabajo investigativo, trataremos en primer lugar una discusión teórica acotada al grado de profesionalización de la comunicación estratégica, expresada en la adopción y uso de diversas tendencias y herramientas de práctica, especialmente de análisis de Big Data, para luego identificar las principales características del estudio. Posteriormente presentaremos los resultados de la investigación y nuestras conclusiones.

\section{Discusión teórica}

Uno de los grandes avances en el campo de la comunicación estratégica se refiere a la incorporación, caracterización, uso y medición de la efectividad de un plan de construcción de imagen y reputación organizacional a través del análisis de grandes volúmenes de información e interacciones de y con stakeholders críticos. Esta estrategia hace referencia al uso de Big Data o base de datos de un gran tamaño y de mayor complejidad que las herramientas usadas habitualmente para el análisis de la información. El uso de los datos que se presenta permite un análisis más detallado de los fenómenos sociales que se están investigando. Gallego Colange (2013, p. 69), define Big Data como

la ciencia que se basa en el tratamiento de grandes volúmenes de información con técnicas matemáticas e informáticas y que permite recoger datos, procesarlos y 
visualizarlos obteniendo una gran velocidad en el análisis, pudiendo anticipar tendencias, con el objetivo de comprender y optimizar ciertos servicios en función del comportamiento del usuario, para satisfacer necesidades tanto en tiempo real, como elaborar estrategias de primer orden en un sector determinado.

En la actualidad y, a nivel latinoamericano, las empresas que más utilizan este tipo de estrategia son aquellas que se dedican a la cotización en la bolsa. También algunas instituciones gubernamentales lideran este tipo de análisis gracias a la enorme disponibilidad de información que poseen desde el procesamiento de datos tributarios. En este sentido, Ortiz, Joyanes y Giraldo (2016), señalan que una de las principales debilidades que tienen medianas empresas de marketing a nivel internacional se relaciona no sólo con la exposición a volúmenes crecientes de información, sino específicamente a la incapacidad manifiesta de aquellos que toman decisiones sobre las estrategias de mercadotecnia para procesar y canalizar la información obtenida. Una buena aproximación analítica a los datos permite identificar patrones de consumo o estrategias óptimas de fidelización de clientes. El gran desafío que advierten estas autoras es que las empresas logren utilizar los crecientes volúmenes de información y su variabilidad para incrementar los ingresos en un mercado cada vez más competitivo y globalizado. Advierten en ese sentido que la tarea para los estrategas de marketing y comunicaciones no es nada fácil, toda vez que los diseños gerenciales pocas veces logran ponderar y alinear los objetivos organizacionales con las necesidades y expectativas del entorno expresados en el uso eficiente del Big Data y en la incorporación de herramientas tecnológicas para su análisis.

Gutiérrez, García y Salas en el año 2016 al realizar una investigación sobre el uso de los Big Data como herramienta de georreferenciación de información, destacan el potencial del uso de esta tecnología, pero advierten que una de las complejidades en ella radica en la capacidad de los ordenadores para procesar grandes volúmenes de información, junto con las competencias que deben tener los analistas para su procesamiento funcional, que va desde el análisis de las redes sociales hasta el estudio de los comportamientos de los consumidores en espacios de georreferenciación. Sobre todo, en un contexto irreversible de ramificación de novedosos formatos de información y comunicación enlazados al uso cotidiano de redes de interacción social digital accesibles desde nuevos dispositivos tecnológicos y móviles.

Los mismos autores citando un estudio de Kitchin (2013) señalan que, al fenómeno de la proliferación de enormes volúmenes de información disponible para los análisis en ciencias sociales, hay que sumar también la velocidad con que esta información se genera, junto con su diversidad y variedad. Además, destacan la naturaleza relacional que puede establecer este tipo de información con otras fuentes gubernamentales oficiales y en especial la flexibilidad desde el punto de vista de la extensión de datos y la escalabilidad gradual de estos. Lo anterior permitiría a la larga estudios más detallados del comportamiento de los consumidores y de un conjunto de actores que afectan los objetivos estratégicos de una organización, conducentes a su vez a la elaboración de estrategias comunicacionales más acertadas.

Por otro lado, Segerberg y Bennett (2011) analizando los contenidos de los tweets a propósito de las protestas contra el cambio climático y la concentración empresarial en 
determinadas industrias, constataron la enorme importancia del análisis de Big Data a la hora de medir e incluso predecir el comportamiento de agrupaciones ciudadanas. Más concretamente, algunas dinámicas conductuales que se establecen en concentraciones de personas en protestas. Por medio de tweets geo localizados estos autores lograron entre otras cosas, identificar los espacios al interior de las principales ciudades europeas utilizados por los manifestantes para la coordinación de sus acciones de protesta.

En una línea similar, Shelton et al. (2015) identificaron fenómenos de agrupación colectiva en ciudades como Río de Janeiro. Esto mediante el uso de herramientas de geolocalización que analizaban el grado de mezcla social en el uso del espacio territorial. Una investigación similar fue la realizada por Mocanu et al. en el año 2013, donde a través de patrones de comportamiento lingüístico asociados al uso de Big Data en tweets lograron identificar fenómenos de diversidad cultural al interior de las ciudades que tomaban como referencia para sus estudios.

Este tipo de investigaciones comprueba que los potenciales de análisis de Big Data son infinitos y no solamente asociables a una georreferenciación, pero también por ejemplo al acceso, caracterización y ponderación con gran precisión de determinados grupos de stakeholders críticos, que afectan y son afectados por los objetivos estratégicos de una empresa (Holtzhausen, 2016).

Dentro de esos stakeholders a abordar, hay quienes incorporan a los social media influencers (aún cuando para efectos prácticos se los tienda a considerar como canales funcionales a la sensibilización de ciertos grupos de interés). Charest y Bouffard (2015) señalan a los social media influencers, así como los defensores de marca, como un nuevo tipo de líderes de opinión y actores a ser conectados e influidos, y cuya visibilidad de opiniones repercute en la reputación de las organizaciones. Quiénes son, qué temas priorizan, cómo y con quién interactúan o qué estrategias y mensajes funcionan para captar su atención y fidelizarlos, son parte de los datos obtenibles a través de un análisis eficiente de Big Data.

Pese al creciente interés que suscitan este tipo de especialistas de las comunicaciones, los estudios muestran que suele existir una falta de comprensión de estos a través de un levantamiento de información pertinente. Adicionalmente a esto, se advierte que las estrategias comunicacionales efectivas por parte de community managers organizacionales o de marca especializados, para utilizar dicha información y llevar a cabo una adecuada interacción con las fuentes de información (Charest \& Bouffard, 2015, p. 3).

Pero no sólo los social media influencers son susceptibles de ser estudiados y abordados a partir de un buen uso de grandes volúmenes de información. También existe un conjunto de grupos y público de interés destacados para las organizaciones y centrales de cara a su relación con la sociedad (Aakhus \& Bzdak, 2015). A través de un análisis oportuno de datos es posible establecer el tipo de relación efectiva con las audiencias (Chung \& Taneja, 2016).

Por último, en un entorno de desconfianza e incertidumbre, un uso dedicado y consistente de Big Data puede habilitar, en cuanto herramienta de comunicación 
estratégica, a la tarea de construir y mantener la confianza de los diversos stakeholders en la organización, generar valor y asegurar la sostenibilidad en el largo plazo. Incluso entrega el poder para evaluar si las acciones diseñadas y ejecutadas por los profesionales de la comunicación estratégica, son consistentes con dicho objetivo, o más bien se guían de forma superficial a conectar estrategias corporativas con la comunicación, o servir sólo de apoyo en la toma de decisiones de altos directivos. La discusión es interesante considerando el caso chileno en cuanto al uso intensivo de las redes sociales, el crecimiento de la interconectividad al interior del territorio, la expansión de la formación universitaria y la creciente inserción del país a través de la globalización económica y el uso de las nuevas tecnologías de información y comunicación. Un punto valioso si incorporamos al análisis los aportes de Castells (2001), cuando remarca el papel de la conectividad gracias a los flujos informativos en una sociedad red.

\section{Método}

El presente estudio utiliza los datos obtenidos del informe del Latin American Communication Monitor 2016-2017, una investigación bianual que analiza las prácticas actuales y el desarrollo futuro de la comunicación estratégica digital en empresas, agencias de comunicación, consultoras y profesionales de la comunicación en Chile y América Latina. Es organizado por la European Public Relations Education and Research Association (EUPRERA), junto a la Asociación de Directivos de Comunicación de España (Dircom) y ejecutado en el caso chileno por el Magíster de Comunicación Estratégica de la Universidad Adolfo Ibáñez.

La investigación aplicó en su segunda edición un cuestionario online que incluyó 33 preguntas agrupadas en 15 secciones. El cuestionario auto administrado se liberó entre los meses de mayo y agosto de 2016. En Latinoamérica la muestra incluyó a más de 900 profesionales.

En el caso de Chile, la muestra consideró a cerca de 90 profesionales de la comunicación de empresas listadas en bolsa de comercio (IPSA), públicas, agencias y consultoras de comunicación estratégica y digital más importantes del país. Estos profesionales en su mayoría provienen de consultoras o agencias de comunicación, así como de empresas públicas y privadas. Cuentan de forma predominante con más de 10 años de experiencia de práctica en la disciplina de la comunicación estratégica. 
Figura 1

\begin{tabular}{|c|c|c|c|}
\hline \multicolumn{4}{|c|}{ Perfil demográfico de los encuestados } \\
\hline Cargo & & Organización & \\
\hline $\begin{array}{l}\text { Director del departamento de } \\
\text { comunicación corporativa u } \\
\text { organizacional / CEO de agencia }\end{array}$ & $33.7 \%$ & $\begin{array}{l}\text { Departamento de comunicación } \\
\text { - Empresa cotiza en bolsa } \\
\text { - Empresa privada }\end{array}$ & \\
\hline Jefe de equipo / Jefe de unidad & $26.3 \%$ & $\begin{array}{l}\text { - Empresa estatal, sector público u organización } \\
\text { política }\end{array}$ & $67.1 \%$ \\
\hline Miembro de equipo / Consultor & $38.3 \%$ & - Organización sin ánimo de lucro o asociación $10.5 \%$ & \\
\hline Otro & $1.8 \%$ & $\begin{array}{l}\text { Agencia, consultoría, relaciones publicas, consultor } \\
\text { freelance }\end{array}$ & $32.6 \%$ \\
\hline Experiencia laboral & & $\begin{array}{l}\text { Alineamiento del departamento de } \\
\text { comunicación }\end{array}$ & \\
\hline Más de 10 años & $49.1 \%$ & $\begin{array}{l}\text { Departamento de comunicación fuertemente } \\
\text { alineado }\end{array}$ & $24.3 \%$ \\
\hline De 6 a 10 años & $26.2 \%$ & Departamento de comunicación alineado & $56.6 \%$ \\
\hline Menos de 5 años & $24.7 \%$ & Departamento de comunicación débilmente alineado & $19.0 \%$ \\
\hline
\end{tabular}

Fuente: Elaboración propia

Los datos obtenidos de la muestra se analizaron estadísticamente utilizando el programa estadístico SPSS versión 24. Además, se recurrió a un análisis de clúster para clasificar a los sujetos en ciertas preguntas de investigación. También se realizó un testeo de relaciones entre componentes de la encuesta y su significancia estadística a través de las pruebas de Chi2, correlación de Pearson, ANOVA/Scheffe Postrhoc, test de independencia de la $\mathrm{T}$ de Kendall y el test de correlación de Kendall.

\section{Resultados}

De los resultados obtenidos se identificó que a nivel general más del $50 \%$ de los profesionales encuestados no han implementado actividades de big data. Son las empresas que cotizan en bolsa y las organizaciones públicas quienes lideran la implantación de big data, mientras que las agencias y consultoras están lejos de este nivel. ¿Cuáles son las razones de este uso tan limitado? Por un lado, casi la mitad de los profesionales asegura que les faltan habilidades analíticas para manejar el uso de altos volúmenes informativos $(45,1 \%) \mathrm{y}$, tiempo para estudiarlos o analizarlos $(35,9 \%)$.

Además, si consideramos la figura 2, se evidencia una mayor importancia asignada al uso del Big Data en equipos profesionales o comunicadores con más experiencia en el mercado laboral. Esta información se relaciona también con los niveles jerárquicos. De esta manera, los directivos de las empresas otorgan más importancia $(48,5 \%)$ a este tipo de fenómeno que los miembros de los equipos de gestión (37,5\%). Esto nos hace presumir que, pese a la importancia dada al uso de altos volúmenes de información, su uso estratégico es mayormente conceptualizado en los altos mandos de las organizaciones. Otro elemento que quizá podría ser abordado en futuras investigaciones hace referencia a los niveles de manejo técnico de la información y las 
estrategias de procesamiento de esta última, en términos de las profesiones y formaciones académicas de los equipos de trabajo relacionados con el área de las comunicaciones.

Figura 2. Nivel de importancia prestado al uso de Big data por rango de edad.

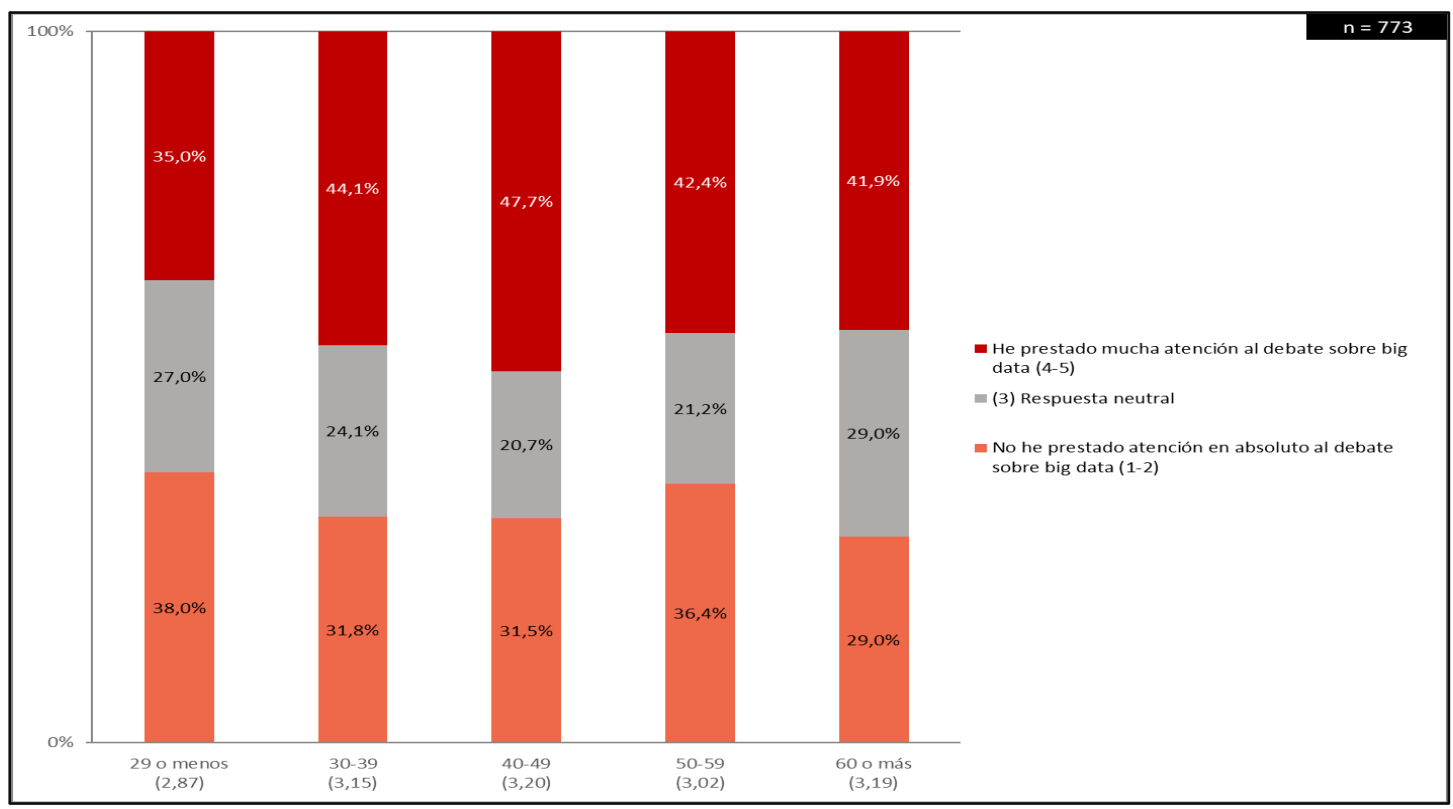

Fuente: Elaboración propia

El cuestionario aplicado a los profesionales de las empresas media también hasta qué punto los comunicadores tenían una comprensión completa del Big Data, incluyendo sus características de volumen, variedad, velocidad y veracidad. De acuerdo con sus respuestas, un análisis descriptivo permitió determinar que más del $50 \%$ de encuestados o prestan atención, pero tienen pocos conocimientos sobre volúmenes considerables de información. Además, se constató que son ajenos al debate y el conocimiento con relación a estos volúmenes informativos. De aquellas organizaciones que lo usan, el 71,2\% lo emplea para planificar las estrategias generales. Algo menos para guiar las acciones del día a día $(58,8 \%)$ y para justificar sus actividades $(53,7 \%)$.

Lo anterior nos permite identificar finalmente que su uso continúa siendo bajo en Chile y en la región en general. La figura 3 nos permite constatar tal fenómeno. 
Figura 3. Implementación de las estrategias de Big data a nivel regional en Latinoamérica.

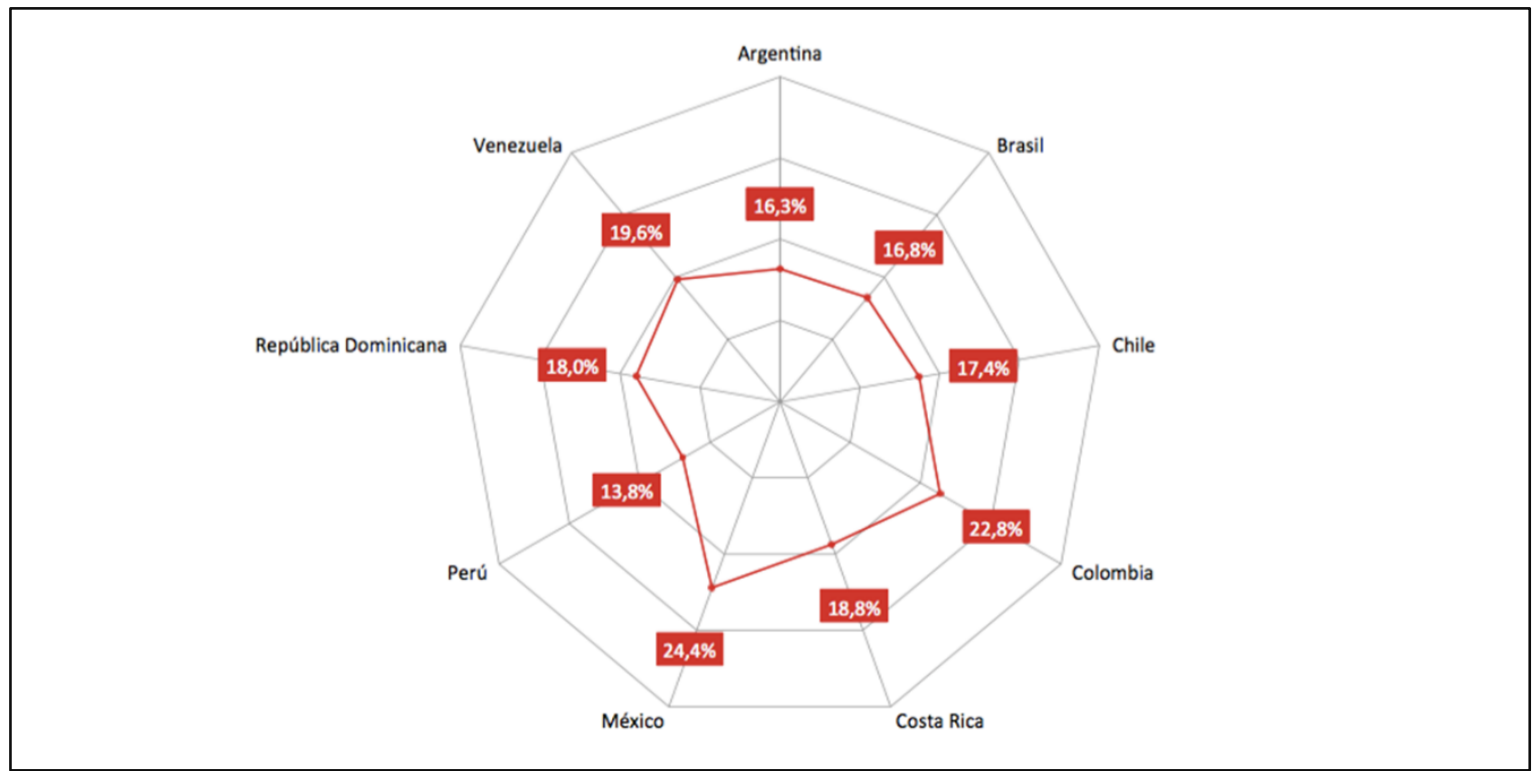

Fuente: Elaboración propia.

A manera de hipótesis para futuras investigaciones pensamos que la baja penetración regional y local en Chile sobre el uso estratégico de la información está relacionada con los niveles de desarrollo y penetración de las nuevas tecnologías de información y comunicación, junto con la realidad técnica y profesional de los recursos humanos existentes al interior de los procesos productivos empresariales.

Se suma a esto la posibilidad de que se sigan privilegiando canales tradicionales de uso y manejo de la información (canales de TV o prensa, junto con el contacto directo). Para el caso chileno, estas hipótesis preliminares son plausibles pero intuitivas a la luz de los resultados presentados, pese a que es necesario que otros estudios lo profundicen.

Pero el análisis de Big Data también es relevante para caracterizar, ponderar y medir nivel y tipo de relacionamiento con determinados grupos de stakeholders críticos, entre ellos, los social media influencers.

Las tres principales plataformas en el mundo - Twitter, blogs y Facebook (Launchmetrics, 2015) - y en Latinoamérica -Facebook, blogs y LinkedIn (ComScore, 2015)- desempeñan un papel de suma importancia como canales para la difusión de información e ideas, además del ejercicio de la influencia de unos usuarios sobre otros. Lo que se constata, sin embargo, es que pese a destacar hoy en día el peso de las redes sociales y el rol de los comunicadores sociales, es plausible pensar que a nivel latinoamericano la inserción de este tipo de cambio cultural es altamente heterogéneo y es posible que este asociado al nivel de penetración no solo de las nuevas tecnologías de información y comunicación sino también, a cambios organizacionales y modos de pensar la comunicación al interior de las empresas y los gobiernos. 
Figura 4. Indicadores importantes para identificar los SMI en los países latinoamericanos

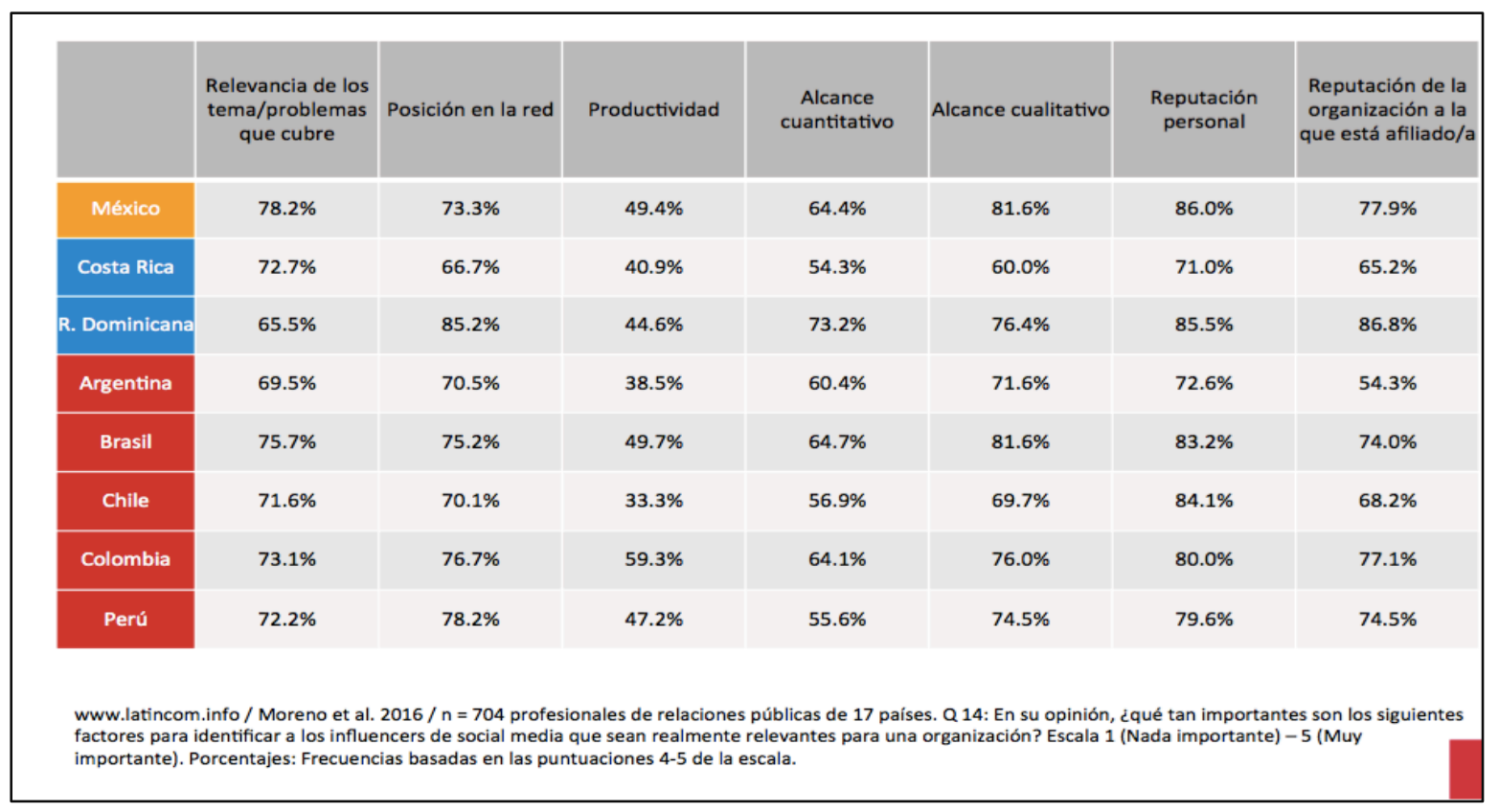

Fuente: Elaboración propia.

Una realidad parecida asoma cuando evaluamos en Chile la identificación y relacionamiento de las organizaciones con una diversidad de stakeholders más allá de los influencers en redes sociales. El engagement de stakeholders, también medible a través del uso de Big Data, refiere a diferentes estados de involucramiento en múltiples canales de comunicación (no sólo las redes sociales). Esto es, estados cognitivos (estar interesado en una organización o marca); afectivos (hablar positivamente de ella) y participativos o posibilidad de un stakeholder de colaborar e influir en la organización (Macnamara, 2016).

Cabe preguntarse entonces, ¿qué entienden por engagement en Chile los profesionales de la comunicación que se desempeñan en agencias, empresas IPSA e instituciones públicas? Los resultados no son muy alentadores. Primero, porque los profesionales de la comunicación chilenos presentan una visión más reduccionista del concepto (tienden a asociar engagement mayoritariamente con niveles de interacción a través de las redes sociales). Y luego, porque una vez lo incorporan en su jerga y actividad diaria, lo hacen de manera deficiente. Menos de la mitad de los encuestados (44,9\%), entienden que un buen engagement implica un trabajo conjunto de los stakeholders y la organización, una comunicación activa y entrega de feedback permanente, y una capacidad de los seguidores y stakeholders de influenciar la toma de decisiones de la organización. Estas brechas pueden estar relacionadas con una falta de competencias laborales y la formación profesional específica que les permita ser proactivos y adaptativos a los cambios o a una identificación de los niveles y características del relacionamiento de la organización con sus diversos stakeholders. Esto último queda ratificado cuando se analizan los datos de la encuesta aplicadas a estos profesionales. A modo de ejemplo, a la pregunta si ha usado herramientas algorítmicas programadas para la adaptación de contenido que puedan conducir a un mejor entendimiento o relacionamiento con stakeholders críticos, del total de la muestra (914 sujetos), solo un 
15,8\% manifestó que su empresa había utilizado esta herramienta. Conocimiento y capacidad para adaptarse a este nuevo enfoque de las comunicaciones podría ser una primera aproximación a esta falta de experticia.

Respecto de la valoración que los profesionales de la comunicación chilenos hacen del concepto de engagement, es interesante observar que no obstante una potencial baja aplicación en el día a día de sus labores sí existe una correcta conceptualización y reconocimiento de su relevancia en varios niveles. En primer lugar, al rol que juegan las motivaciones y el interés como factores claves de un engagement exitoso. En efecto, a la afirmación engagement significa estar interesado o sentirse involucrados, en una escala de 1 a 5 siendo la primera totalmente en desacuerdo y la quinta totalmente de acuerdo, la muestra se inclina hacia esta última con un 4,12. Asimismo y en un punto similar, a la afirmación engagement significa estar entusiasmado, orgulloso o vinculado emocionalmente, en la misma escala, los entrevistados se inclinaron en promedio en torno a un 4,16. Lo anterior es interesante porque identifica una valoración de dimensiones no solo de naturaleza cognitiva, pero también afectivas. Es fundamental aquí precisar que otras investigaciones podrían profundizar estos hallazgos. Pero nos parece importante resaltarlos considerando la relevancia que los profesionales de la comunicación y la gestión del cambio otorgan a variables como la motivación y la implicancia afectiva, pilares de una moderna gestión al interior de las empresas.

Ahora bien, al analizar los datos obtenidos de la encuesta aplicada a los profesionales chilenos, constatamos que, pese a que estos están vinculados a las comunicaciones, valoran de manera importante y significativa las nuevas formas de comunicación, pero las competencias relacionadas a los social media siguen siendo bajas. Esto se sustenta con la información que se presenta en la tabla 1.

Tabla 1. Competencias en social media de los profesionales de las comunicaciones en Chile.

\begin{tabular}{lr}
\hline & Puntuación media \\
Distribución de mensajes via social media & 3,06 \\
Conocimiento sobre las tendencias de social media & 2,98 \\
Conocimiento de cómo evitar riesgos y manejar crisis en social media & 2,99 \\
Desarrollo de estrategias de social media & 2,83 \\
Identificación de influencers en social media & 2,92 \\
Inicio de diálogo online con stakeholders & 2,88 \\
Evaluación de las actividades de social media & 2,82 \\
Interpretación de datos de monitoreo de social media & 2,85 \\
Gestión de comunidades online & 2,74 \\
Configuración de plataforma de social media & 2,40 \\
Conocimiento del marco legal para social media & 2,21 \\
Comprensión del uso de algoritmos (ej. Usando plataformas de social media) & 2,12 \\
\hline
\end{tabular}

Fuente: Elaboración propia en base a www.latin.com [Escala de 1 a 5 donde 1 es baja y 5 muy alta]

Estos datos ratifican las apreciaciones de Killingsworth, C., \& Flynn, T. (2016), sobre el papel de los comunicadores y sus habilidades en el escenario contemporáneo. Esto contrasta con las proyecciones que a mediados de los años 200 manifestaba Castells 
(2005), sobre el desarrollo tecnológico y los efectos de la globalización para el caso chileno. No obstante, los avances, el uso estratégico del Big Data para los profesionales de las comunicaciones sigue en un rezago, poniendo un desafío no solo a la formación profesional al interior de la Educación Terciaria (Freberg, K., \& Kim, C. M., 2017) sino a la capacitación y formación permanente al interior de las organizaciones empresariales. Se suma a esto dos conclusiones preliminares.

La primera es sobre el tipo de competencias que las instituciones de Educación Superior (principalmente universitaria), desarrollan orientadas a los nuevos mercados y a las tecnologías de comunicación e información. La segunda, el tipo de entorno empresarial, los soportes tecnológicos y la infraestructura que permita el desarrollo de competencias profesionales. El desafío aquí no solo está en el uso intensivo de la web para la comunicación y la transmisión de datos, sino en el uso estratégico que se puede dar en concordancia con mercados cada vez más competitivos en donde la innovación y el uso de grandes bases de datos requieren.

Pese a la importancia dada a la comunicación online vía webs, e-mail, intranets en su proyección hacia el futuro (ver tabla 2), los datos comprueban los desafíos en la formación de competencias actuales para los profesionales de las comunicaciones al interior del país.

Tabla 2. Importancia en 2019 dada a la comunicación online vía webs, e-mail, intranets.

\begin{tabular}{lcc}
\hline & Frecuencia & Porcentaje \\
Nada importante & 1 & 1,1 \\
Poco importante & 7 & 7,4 \\
Importante & 4 & 4,2 \\
Muy importante & 20 & 21,1 \\
Extremadamente importante & 38 & 40 \\
No sabe/no contesta & 25 & 26,3 \\
Total & 95 & 100 \\
\hline
\end{tabular}

Fuente: Elaboración propia. 
Figura 5. Los indicadores predilectos para detectar el engagement con stakeholders en Latinoamérica

\begin{tabular}{|c|c|c|c|c|c|c|}
\hline México & $37.5 \%$ & $40.6 \%$ & $64.6 \%$ & $33.3 \%$ & $59.4 \%$ & $46.9 \%$ \\
\hline Costa Rica & $62.0 \%$ & $50.0 \%$ & $66.0 \%$ & $40.0 \%$ & $30.0 \%$ & $32.0 \%$ \\
\hline Argentina & $30.9 \%$ & $61.8 \%$ & $62.7 \%$ & $42.7 \%$ & $57.3 \%$ & $32.7 \%$ \\
\hline Brasil & $40.2 \%$ & $56.3 \%$ & $51.7 \%$ & $33.9 \%$ & $52.9 \%$ & $44.8 \%$ \\
\hline Chile & $35.8 \%$ & $54.7 \%$ & $52.6 \%$ & $43.2 \%$ & $57.9 \%$ & $36.8 \%$ \\
\hline Colombia & $38.0 \%$ & $52.8 \%$ & $56.3 \%$ & $48.6 \%$ & $59.2 \%$ & $31.7 \%$ \\
\hline
\end{tabular}

Fuente: Elaboración propia.

Por último, la investigación obtenida permite evidenciar que el uso de Big Data, influencers o estrategias de engagement con públicos relevantes en cuanto elementos centrales de una comunicación estratégica, son percibidos por los profesionales de la comunicación en Chile como una vía para conectar estrategias corporativas con la comunicación (no en influirlas), apoyar la toma de decisión de sus jefes o, enfrentarse con mayores instrumentos a la marea digital y plataformas sociales de moda. Pero poco o nada con fortalecer y mejorar la confianza en la organización.

Figura 6. Relevancia percibida de los temas estratégicos en Latinoamérica

\begin{tabular}{|c|c|c|c|c|c|c|c|}
\hline & \begin{tabular}{c|} 
Conectar las \\
estrategias de la \\
organización con \\
la comunicación
\end{tabular} & \begin{tabular}{|c|} 
Lidiar con la \\
velocidad y el \\
volumen del flujo \\
de información
\end{tabular} & $\begin{array}{c}\text { Enfrentarse con } \\
\text { la evolución } \\
\text { digital y la web } \\
\text { social }\end{array}$ & $\begin{array}{c}\text { Fortalecer el rol de la función } \\
\text { de comunicación para apoyar } \\
\text { la toma de decisiones de los } \\
\text { altos directivos }\end{array}$ & $\begin{array}{c}\text { Construir y } \\
\text { mantener la } \\
\text { confianza en } \\
\text { la } \\
\text { organización }\end{array}$ & $\begin{array}{l}\text { Usar big data y/o } \\
\text { algoritmos para } \\
\text { la comunicación }\end{array}$ & $\begin{array}{l}\text { Habilitar, hacer } \\
\text { coaching y } \\
\text { aconsejar a los } \\
\text { directivo }(s) \text { sénior } \\
\text { y otros empleados }\end{array}$ \\
\hline México & $34.4 \%$ & $37.5 \%$ & $47.9 \%$ & $34.4 \%$ & $18.8 \%$ & $35.4 \%$ & $11.5 \%$ \\
\hline Costa Rica & $38.0 \%$ & $26.0 \%$ & $54.0 \%$ & $36.0 \%$ & $14.0 \%$ & $36.0 \%$ & $6.0 \%$ \\
\hline R. Dominicana & $31.7 \%$ & $20.6 \%$ & $50.8 \%$ & $25.4 \%$ & $28.6 \%$ & $42.9 \%$ & $9.5 \%$ \\
\hline Argentina & $30.9 \%$ & $40.0 \%$ & $33.6 \%$ & $30.0 \%$ & $17.3 \%$ & $40.9 \%$ & $13.6 \%$ \\
\hline Brasil & $29.3 \%$ & $43.7 \%$ & $30.5 \%$ & $28.7 \%$ & $19.5 \%$ & $32.8 \%$ & $12.6 \%$ \\
\hline Chile & $40.0 \%$ & $26.3 \%$ & $35.8 \%$ & $38.9 \%$ & $25.3 \%$ & $26.3 \%$ & $6.3 \%$ \\
\hline Colombia & $23.9 \%$ & $29.6 \%$ & $37.3 \%$ & $50.0 \%$ & $27.5 \%$ & $40.1 \%$ & $16.2 \%$ \\
\hline Perú & $43.8 \%$ & $28.1 \%$ & $34.4 \%$ & $43.8 \%$ & $17.2 \%$ & $37.5 \%$ & $14.1 \%$ \\
\hline Venezuela & $28.3 \%$ & $35.8 \%$ & $41.5 \%$ & $37.7 \%$ & $18.9 \%$ & $58.5 \%$ & $57.0 \%$ \\
\hline
\end{tabular}

Fuente: Elaboración propia. 
El distanciamiento con una auténtica comunicación estratégica también se observa el evaluar los canales de comunicación privilegiados por los profesionales. La mayoría dice utilizar las redes sociales (cerca de un $70 \%$ ) y pocos $(38 \%)$ trabajan un relacionamiento a partir de medios propios (menos costosos y susceptibles de un mayor control de lo que se comunica) o ganados (como influencers sociales no pagados que puedan certificar a la marca o institución).

Se suma a lo anterior la capacidad que manifiestan para adaptarse a los cambios y a la innovación. De la muestra total de profesionales, un $64,8 \%$ manifiesta que es capaz de adaptarse a estos, lo que más bien sería una pregunta que apunta a un cierto nivel de deseabilidad social, pero que no necesariamente se relaciona con capacidades observables en la gestión del cambio comunicacional gracias al uso de Macrodatos. Es probable que como afirma Tascón (2013), la complejidad de un dominio profundo sobre el uso y gestión de altos volúmenes de información se deba a la complejidad de la materia que además de lo señalado anteriormente se suman elementos relacionados con conocimientos computacionales, vinculados con minería de datos o inteligencia de negocios (Business intelligence).

La realidad evidenciada abre una ventana para avanzar no sólo en una mayor profesionalización de las comunicaciones. Pero sobre todo para superar culturas, estrategias y formas de relacionamiento que sólo sostienen o detienen una mejora en los niveles de confianza hacia empresas, empresarios, marcas e instituciones públicas de gran relevancia y aporte a la sociedad.

\section{Conclusión}

La presente investigación de cuenta de un fenómeno de Big Data poco estudiado a nivel regional y local. El alto nivel de información disponible en la web nos permite intuitivamente establecer que, gracias a estos volúmenes de datos y su correcto y oportuno análisis, las decisiones a nivel corporativo pueden ser más eficaces a la hora de evaluar por ejemplo los niveles de productividad o la capacidad para construir relaciones de confianza y duraderas con determinados públicos de interés. Sin embargo, la evidencia mostrada revela un uso precario de este tipo de herramientas.

No cabe duda de que el uso del Big Data abre nuevos desafíos. Por un lado, la capacidad de procesamiento y comprensión de altos flujos de información, no solo para el desarrollo de la industria de la informática, sino también para los profesionales encargados del procesamiento e interpretación de estos flujos y concentraciones de datos. Estos requieren habilidades de análisis que en muchas oportunidades y a la luz del análisis de los datos y la revisión de la literatura, carecen o no van de la mano con el desarrollo y las demandas que la sociedad requiere. Por otro lado, se encuentra dimensión ética. En esto pensamos principalmente en el uso de la información que muchas veces es de carácter privada, no obstante, la necesidad de transparencia en la sociedad contemporánea (Rosanvallon, 2007; Han, 2013). Hay, no obstante, este último otro elemento que es llamativo al interior del debate sobre el uso de Big Data y es el tema del control sobre la sociedad, lo que no solamente nos dice sobre qué es lo que efectivamente se hace con la 
información reservada de los sujetos, sino que también con qué propósito se almacenan los datos y cómo esto puede afectar a los gobiernos, a las empresas y a las personas.

Lo anterior nos permite reflexionar sobre las formas de conexión e interacción al interior de la sociedad definida quizá ya como sociedad red (Castells, 2009). Vinculando estas reflexiones con nuestra pregunta inicial de investigación, pensamos que una de las habilidades centrales de los encargados de la gestión de la información se encuentra no solamente con generar adecuados procesos o procedimientos de almacenamiento y uso eficiente de la información, sino también la capacidad de lograr influir en el escenario mediático que las redes sociales generan. En este sentido, las habilidades sobre el tratamiento de Macrodatos (Big Data), se relacionan a nuestro juicio con la posibilidad de generar nuevos flujos informativos, saber comunicarlos y disuadir al interior de la esfera mediática.

Los desafíos descritos se insertan en un contexto donde al interior de las empresas se requieren menores costos en tiempo y dinero para el tratamiento de la información. Además de esto, las organizaciones empresariales requerirán transparentar la información al interior de ellas para así empoderar, responsabilizar y legitimar las decisiones corporativas en un contexto de permanentes exigencias por participación en la toma de decisiones por parte de los profesionales de las empresas. Se suma a esto un desafío no menor como es el de procesar información donde muchas veces esta se encuentra dispersa y escasamente estructurada (Monleón, 2015). Lo último pone el acento en la capacidad de las organizaciones empresariales de generar entornos donde se promueva el desarrollo de competencias tales como la búsqueda e información, la contrastación entre fuentes y la verosimilitud de estas, elementos que son cruciales para los profesionales encargados de la comunicación (Bradshaw, 2011).

Por otro lado, el uso tal herramienta está relacionada no solo con el proceso productivo o, las relaciones que se tienen con los mercados, pero sobre todo con una dimensión estratégica de negocios que abraza conceptos como la construcción de reputación y sostenibilidad. Pese a que Chile es uno de los países de la región que ha apostado por la liberalización de los mercados y por el uso masificado de las nuevas tecnologías de comunicación e información, los desafíos que ponen escenarios económicos donde se apuesta por la innovación y el cambio son centrales, están dejando atrás al país, pero particularmente sus entornos organizacionales, tanto públicos como privados. Advertimos aquí que, los profesionales encuestados reconocen el valor del uso de altos niveles de información, pero identifican como amenazas las culturas organizacionales en donde se inscriben sus desempeños. Al parecer, y podría ser objeto de otras investigaciones, sería importante indagar por los escenarios posibles de cambio cultural al interior de las Burocracias públicas como privadas que promuevan el uso intensivo de grandes volúmenes de Información con el fin de influir y lograr las metas que las propias organizaciones se hayan trazado.

En esa línea, y pese a ser valorada, la Big Data en términos de los agentes de comunicación y cambio, está al debe como estrategia de posicionamiento de las firmas. A los usos y estrategias habituales de comunicación, se debe sumar la distribución del tiempo al interior de los entornos empresariales. Pese a que no fue abordado por la 
investigación, parece relevante indagar también por el tipo de formación profesional al interior del mercado de Educación Terciaria existente en Chile, junto con pesquisar por el tipo de entorno empresarial y los reales soportes tecnológicos que permitan el desarrollo de competencias relacionadas con la innovación y el uso de grandes bases de información. Por otro lado, y junto con esto y a modo de hipótesis para futuros estudios, consideramos se debe profundizar en los niveles de capacitación permanente y competencias técnicas específicas por parte de los profesionales de la comunicación a nivel local a cargo del uso de Big Data. Asociado a esto y tema no menor es la incorporación de tecnología que permita el procesamiento de altos volúmenes de información. Pese a que no fue parte de esta investigación, consideramos que esta es una línea interesante de ser abordada, ya que avanzaría en un mejor entendimiento y perfeccionamiento de la relación existente entre cambio paradigmático de gestión de la información, con la toma de decisiones a nivel directivo. Esta toma de decisiones podría estar identificada con un indicador de cambio organizacional a nivel de actualización tecnológica, junto con el desarrollo de competencias para los equipos de profesionales.

La realidad evidenciada abre una ventana para avanzar no sólo en una mayor profesionalización de las comunicaciones, sino también para superar culturas, estrategias y formas de relacionamiento que sólo sostienen o detienen una mejora en los niveles de confianza hacia empresas, empresarios, marcas e instituciones públicas de gran relevancia y aporte a la sociedad.

\section{Referencias}

Anand, B. (2016). The content trap: A strategist's guide to digital change. Random House. Publishing Group.

Blanco, T. P., \& Herrera, J. S. (2014). Nuevas tendencias en comunicación estratégica. Madrid: ESIC Editorial.

Bradshaw, P. (2011). Data Journalism. Recuperado de: https://onlinejournalismblog.com/2011/07/07/the-inverted-pyramid-of-data-journalism/

Manuel, C. (2009). Comunicación y poder. Barcelona: Editorial Alianza.

Castells, M. (2008). Comunicación, poder y contrapoder en la sociedad red (I). Los medios y la política. Telos: Cuadernos de Comunicación E Innovación 74:13-24.

Castells, M. (2005). Globalización, desarrollo y democracia: Chile en el contexto mundial. Santiago: Fondo De Cultura Económica.

Castells, M. (2001). La era de la Información: economía, sociedad y cultura: la sociedad red. Madrid: Alianza.

Charest, F., \& Bouffard, J. (2015). The characteristics of the e-influence of Community Managers: Issues for the e-reputation of organizations. Public Relations Review, 41(2), 302-304. 
Calonge, F. G. (2013). Twitter y Televisión. El papel de la gestión analítica de las audiencias sociales. Big Data, 95, 68-76. Recuperado de: https://telos.fundaciontelefonica.com/docs/2013/11/11/11400001_4_4_0.pdf

Gutiérrez-Puebla, J., García-Palomares, J. C., \& Salas-Olmedo, M. H. (2016). Big (Geo) Data en Ciencias Sociales: Retos y Oportunidades. Revista de Estudios Andaluces, 33(1),1-23. http://dx.doi.org/10.12795/rea.2016.i33

Freberg, K., \& Kim, C. M. (2017). Social Media Education: Industry Leader Recommendations for Curriculum and Faculty Competencies. Journalism \& Mass Communication Educator. doi.org/10.1177/1077695817725414

Han, B. C. (2013). La sociedad de la transparencia. Barcelona: Herder Editorial.

Holtzhausen, D. (2016). Datafication: threat or opportunity for communication in the public sphere? Journal of Communication Management, 20(1), 21-36. doi.org/10.1108/JCOM-12-2014-0082

Ihm, J., \& Hsieh, Y. P. (2015). The implications of information and communication technology use for the social well-being of older adults. Information. Communication \& Society, 18(10), 1123-1138. doi: 10.1080/1369118X.2015.1019912

Kitchin, R. (2013). Big Data and human geography Opportunities, challenges and risks2, Dialogues in Human Geography, 3(3), 262-267. doi.org/10.1177/2043820613513388

Killingsworth, C., \& Flynn, T. (2016). Assessing the CPRS Pathways to the Profession competency framework: Perspectives on corporate communication leadership competencies and credentials. Corporate Communications: An International Journal, 21(2), 177-194. doi.org/10.1108/CCIJ-03-2015-0013

Launchmetrics (2015). Estatus y prácticas de las relaciones con Influencers. Recuperado de: $\quad$ https://www.launchmetrics.com/es/recursos/whitepapers/informeinfluencers-2015

Mocanu, D., Baronchelli, A., Perra, N., Gonçalves, B., Zhang, Q. y Vespignani, A. (2013): "The twitter of babel: Mapping world languages through microblogging platforms", PloS One, 8(4), e61981. doi.org/10.1371/journal.pone.0061981

Monleón-Getino, A. (2015). El impacto del Big-data en la Sociedad de la Información. Significado y utilidad. Historia y Comunicación Social, 20(2), 427445.doi.org/10.5209/rev_HICS.2015.v20.n2.51392

Morales, M. D. O., Aguilar, L. J., \& Marín, L. M. G. (2016). Los desafíos del marketing en la era del big data. Revista e-Ciencias de la Información, 6(1), 1-30, http://dx.doi.org/10.15517/eci.v6i1.19005

Pieczka, M. (2015). Dialogue and critical public relations. The Routledge Handbook of Critical Public Relations. Oxon: Routledge. 
Rosanvallon, P. (2007). La contrademocracia: la política en la era de la desconfianza. Buenos Aires: Editorial Manantial.

Shelton, T., Poorthuis, A., \& Zook, M. (2015). Social media and the city: Rethinking urban socio-spatial inequality using user-generated geographic information. Landscape and Urban Planning, 142, 198-211.

Tascón, M. (2013). Pasado, presente y futuro. Big Data. Cuadernos de Comunicación e Innovación. Telos 95, 47-50.

Takhteyev, Y., Gruzd, A., \& Wellman, B. (2012). Geography of Twitter networks. Social Networks, 34(1), 73-81. http://dx.doi.org/10.1016/j.socnet.2011.05.006

\section{Notas}

1 Juan Cristóbal Portales Echeverría es Periodista Universidad Católica de Chile, MSc Political Communication London School of Economics and Political Science y PhD Comunicación Política Universidad Complutense de Madrid. Docente, Investigador y Director Magíster de Comunicación Estratégica Universidad Adolfo Ibáñez, Chile. Ha sido asesor de varios gobiernos, campañas políticas y empresas en Chile, Colombia, Ecuador, República Dominicana y Haití. Cuenta con numerosas publicaciones académicas y colaboraciones editoriales en el área de la comunicación estratégica, política, financiera y ASG (Ambiental, Social y de Gobiernos Corporativos). https://orcid.org/0000-0002-2112$\underline{1501}$

2 Juan Alberto Castañeda Alcaíno es Cientista Político Universidad Católica de Chile, Magíster en Estudios Sociales y Políticos Latinoamericanos U. Alberto Hurtado, Doctor (C) en Sociología por la misma universidad. Docente, Investigador U. Católica Silva Henríquez, Chile. Ha sido docente desde hace más de 10 años. Sus áreas de estudio son Sociología Política, Opinión Pública, Confianza Institucional y Metodología de la Investigación Cuantitativa y Cualitativa entre otras. https://orcid.org/0000-0002-2910$\underline{6816}$ 